\title{
Endovascular treatment for acute ischemic stroke in patients with versus without atrial fibrillation: a matched-control study
}

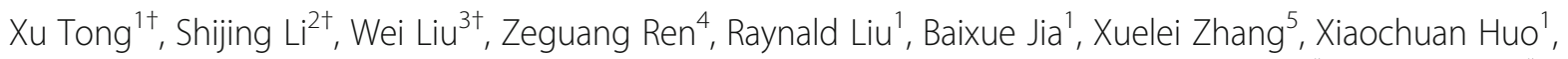
Gang Luo ${ }^{1}$, Gaoting Ma ${ }^{1}$, Anxin Wang ${ }^{6}$, Yilong Wang ${ }^{7}$, Yongjun Wang ${ }^{6}$, Zhongrong Miao ${ }^{1 *}$, Dapeng Mo ${ }^{*}$ and on behalf of ANGEL-ACT study group

\begin{abstract}
Background and objective: The effect of atrial fibrillation (AF) on outcomes of endovascular treatment (EVT) for acute ischemic stroke (AIS) is controversial. This study aimed to investigate the association of AF with outcomes after EVT in AIS patients.
\end{abstract}

Methods: Subjects were selected from ANGEL-ACT registry (Endovascular Treatment Key Technique and Emergency Work Flow Improvement of Acute Ischemic Stroke) - a prospective consecutive cohort of AIS patients undergoing EVT at 111 hospitals in China between November 2017 and March 2019, and then grouped according to having a history of AF or not. After 1:1 propensity score matching, the outcome measures including the 90-day modified Rankin Scale (mRS) score, successful recanalization after final attempt, symptomatic intracranial hemorrhage (ICH) within $24 \mathrm{~h}$, and death within 90 days were compared.

Results: A total of 1755 patients, 550 with AF and 1205 without AF, were included. Among 407 pairs of patients identified after matching, no significant differences were found in the mRS score (median: 3 vs. 3 points; $P=0.29$ ), successful recanalization (87.2 vs. 85.3\%; $P=0.42)$, symptomatic ICH (9. 4 vs. 9.1\%; $P=0.86)$ and death (16.3 vs. 18.4\%; $P=0.44$ ) between patients with and without AF.

Conclusion: The findings of this matched-control study show comparable outcomes of EVT in Chinese AIS patients with and without AF, which do not support withholding EVT in patients with both AIS and AF.

Trial registration: NCT03370939

First registration date: 28/09/2017

First posted date: 13/12/2017

Keywords: Atrial fibrillation, Endovascular treatment, Ischemic stroke, Propensity score matching

\footnotetext{
*Correspondence: zhongrongm@163.com; bjttmodp@163.com

${ }^{+} X u$ Tong, Shijing Li and Wei Liu contributed equally to this work.

'Department of Interventional Neuroradiology, Beijing Tiantan Hospital,

Capital Medical University, No.119 South 4th Ring West Road, Fengtai District, Beijing, China

Full list of author information is available at the end of the article
}

(c) The Author(s). 2021 Open Access This article is licensed under a Creative Commons Attribution 4.0 International License, which permits use, sharing, adaptation, distribution and reproduction in any medium or format, as long as you give appropriate credit to the original author(s) and the source, provide a link to the Creative Commons licence, and indicate if changes were made. The images or other third party material in this article are included in the article's Creative Commons licence, unless indicated otherwise in a credit line to the material. If material is not included in the article's Creative Commons licence and your intended use is not permitted by statutory regulation or exceeds the permitted use, you will need to obtain permission directly from the copyright holder. To view a copy of this licence, visit http://creativecommons.org/licenses/by/4.0/. The Creative Commons Public Domain Dedication waiver (http://creativecommons.org/publicdomain/zero/1.0/) applies to the data made available in this article, unless otherwise stated in a credit line to the data. 


\section{Introduction}

Atrial fibrillation (AF), as the most common cause of cardioembolic stroke, is associated with a 4-5 times increased risk of acute ischemic stroke (AIS) and accounts for approximately $30-40 \%$ of all acute large vessel occlusion (LVO) [1-8]. Patients with AF-related stroke are older, have greater burden of comorbidities and worse neurological deficits, thus have a higher probability of disability or mortality after usual care [9-12]. Furthermore, intravenous thrombolysis (IVT) is less effective on both recanalization and clinical outcome but also increases the risk of intracranial hemorrhage $(\mathrm{ICH})$ in patients with AF. The poor response to IVT could be partly explained by the pathophysiology of AF-related stroke, such as the gaps between patients with and without AF in terms of embolic size and components, collateral status, infarct core volume, and stroke progression [13, 14].

Endovascular treatment (EVT) represented by mechanical thrombectomy with stent-retriever or aspiration catheter has become the standard treatment for selected patients with AIS due to intracranial proximal LVO [15]. However, limited data and conflicting results exist regarding the role of AF on procedural and clinical outcomes after EVT [16-21]. To address this issue and on the hypothesis that the modification of AF was attributed to the effect of case mix; in other words, AF might not independently affect any outcome in EVT-treated patients after adjusting for possible confounders. We therefore performed a matched-control analysis based on a prospective nationwide registry database to assess whether the technical success and functional outcomes differ in LVO patients with and without AF after receiving EVT.

\section{Methods}

\section{Study population}

Data were extracted from ANGEL-ACT (Endovascular Treatment Key Technique and Emergency Work Flow Improvement of Acute Ischemic Stroke), a prospective nationwide registry of 1793 consecutive patients with AIS caused by LVO undergoing EVT in 111 hospitals in China between November 2017 and March 2019. Full methods of the registry, such as inclusion/exclusion criteria and data collection standards, have been reported earlier [22]. The protocol was approved by the ethics committees of all centers, and all participants (or legal representatives) provided written informed consent. The study procedures were in accordance with the 1964 Helsinki declaration and its later amendments.

In this analysis, patients with missing baseline or procedure data in Table 1 were excluded, and the remainder cases were divided into two groups based on whether they had pre-existing AF, identified by previous medical records.

\section{Outcome measures}

The primary outcome was the 90-day modified Rankin Scale (mRS) score assessed by trained and independent investigators. The secondary outcomes included successful recanalization (modified Thrombolysis in Cerebral Infarction [mTICI] of 2b-3) after first and final attempt, complete recanalization (mTICI of 3 ) after final attempt, [23] the proportions of mRS $0-1,0-2$ and $0-3$ at 90 days. The safety outcomes were intra-procedural complications (e.g., new territorial embolization, arterial perforation, arterial dissection, vasospasm requiring treatment and in-stent thrombosis), any $\mathrm{ICH}$, parenchymal hematoma type 2 ( $\mathrm{PH} 2)$ and symptomatic $\mathrm{ICH}$ within 24 hours according to the Heidelberg Bleeding Classification, [24] and death within 90 days.

\section{Statistical analysis}

Data were displayed as median (interquartile range [IQR]) or frequency (percentage). Univariable comparisons of baseline characteristics between patients with and without AF were performed using Mann-Whitney or Pearson's chi-square tests. To improve the comparability between the two groups, a 1:1 propensity score matching (PSM) was performed by using a caliper distance of 0.05 [25]. For comparing the outcomes between both groups, the odds ratios (OR) or common OR with their 95\% confidence intervals (CI) were calculated using a binary or ordinal logistic regression model, if applicable. Significance level was set to $\alpha=0.05$ (2-sided). Statistical analyses were conducted with SAS software version 9.4 (SAS Institute Inc., Cary, NC).

\section{Results}

Among 1793 patients enrolled in the ANGEL-ACT registry, 38 patients were excluded due to missing baseline or procedure information, a total of 1755 patients were included in this analysis, including 550 cases with AF and 1205 without AF. After PSM, 814 patients were identified (Fig. 1).

As shown in Table 1, there were significant differences in many baseline and procedure characteristics between pre-matched patients with and without AF. For example, patients with AF were 8 years older, had 3 points higher NIHSS scores, were more frequently given anticoagulants before stroke onset, and received more passes of thrombectomy than those without AF; while patients with AF had lower proportions of male, current smoker, and vertebro-basilar artery occlusion, were less often given tirofiban during the procedure and emergency angioplasty/stenting, and experienced $65 \mathrm{~min}$ shorter onset-to-puncture time than those without AF (all Pvalues <0.01). After PSM, all baseline and procedure characteristics between groups were well-balanced (Table 1). 
Table 1 Baseline and procedure characteristics of patients with AF versus without AF

\begin{tabular}{|c|c|c|c|c|c|c|c|c|}
\hline \multirow[t]{2}{*}{ Baseline and procedure variables } & \multicolumn{4}{|c|}{ Pre-matched population $(n=1755)$} & \multicolumn{4}{|c|}{ Post-matched population $(n=814)$} \\
\hline & $\begin{array}{l}\text { With AF } \\
(n=550)\end{array}$ & $\begin{array}{l}\text { Without AF } \\
(n=1205)\end{array}$ & $\begin{array}{l}\text { SD } \\
(\%)\end{array}$ & $\overline{P \text {-value }}$ & $\begin{array}{l}\text { With AF } \\
(n=407)\end{array}$ & $\begin{array}{l}\text { Without AF } \\
(n=407)\end{array}$ & $\begin{array}{l}\text { SD } \\
(\%)\end{array}$ & $P$-value \\
\hline Age, median (IQR), years & $71(64-78)$ & $63(54-70)$ & 72.0 & $<0.01$ & $69(62-76)$ & $68(61-75)$ & 4.3 & 0.32 \\
\hline Male sex & $246(44.7)$ & $910(75.5)$ & 66.2 & $<0.01$ & $213(52.3)$ & $221(54.3)$ & 3.9 & 0.57 \\
\hline History of hypertension & $333(60.6)$ & $673(55.9)$ & 9.5 & 0.07 & $232(57.0)$ & $245(60.2)$ & 6.5 & 0.35 \\
\hline History of diabetes mellitus & $99(18.0)$ & $225(18.7)$ & 1.8 & 0.74 & $73(17.9)$ & $82(20.2)$ & 5.6 & 0.42 \\
\hline Prior ischemic stroke & $130(23.6)$ & $207(17.2)$ & 16.1 & $<0.01$ & $85(20.9)$ & $88(21.6)$ & 1.8 & 0.80 \\
\hline Pre-stroke $\mathrm{mRS}$ score $\geq 1$ & $84(15.3)$ & $146(12.1)$ & 9.2 & 0.07 & $55(13.5)$ & $60(14.7)$ & 3.5 & 0.61 \\
\hline Cigarette smoking & & & 56.5 & $<0.01$ & & & 7.3 & 0.27 \\
\hline Never Smoker & $420(76.4)$ & $629(52.2)$ & & & 291 & 285 & & \\
\hline Ex-smoker & $44(8.0)$ & $89(7.4)$ & & & 37 & 28 & & \\
\hline Current smoker & $86(15.6)$ & $487(40.4)$ & & & 79 & 94 & & \\
\hline Systolic blood pressure, median (IQR), mmHg & $\begin{array}{l}145(130- \\
160)\end{array}$ & $\begin{array}{l}145(132- \\
162)\end{array}$ & 7.5 & 0.21 & $\begin{array}{l}145(130- \\
160)\end{array}$ & $\begin{array}{l}145(130- \\
160)\end{array}$ & 2.1 & 0.95 \\
\hline NIHSS score, median (IQR) & $18(14-22)$ & $15(11-21)$ & 29.5 & $<0.01$ & $17(13-21)$ & $17(13-22)$ & 2.6 & 0.87 \\
\hline ASPECTS, median (IQR) ${ }^{\text {a }}$ & $10(7-10)$ & $9(7-10)$ & 13.1 & $<0.01$ & $10(7-10)$ & $10(7-10)$ & 1.5 & 0.91 \\
\hline Occlusion site & & & 46.4 & $<0.01$ & & & 9.1 & 0.20 \\
\hline Internal carotid artery & $166(30.2)$ & $279(23.2)$ & & & $111(27.3)$ & $116(28.5)$ & & \\
\hline Middle cerebral artery M1 segment & $266(48.4)$ & $493(40.9)$ & & & $197(48.4)$ & $187(45.9)$ & & \\
\hline Middle cerebral artery M2 segment & $59(10.7)$ & $91(7.6)$ & & & $47(11.6)$ & $39(9.6)$ & & \\
\hline Vertebro-basilar artery & $49(8.9)$ & $313(26.0)$ & & & $42(10.3)$ & $60(14.7)$ & & \\
\hline Other intracranial arteries ${ }^{b}$ & $10(1.8)$ & $29(2.4)$ & & & $10(2.5)$ & $5(1.2)$ & & \\
\hline Prior use of antiplatelet agents & $101(18.4)$ & $187(15.5)$ & 7.8 & 0.14 & $75(18.4)$ & $73(17.9)$ & 1.3 & 0.86 \\
\hline Prior use of anticoagulants & $51(9.3)$ & $20(1.7)$ & 34.0 & $<0.01$ & $19(4.7)$ & $15(3.7)$ & 4.9 & 0.48 \\
\hline Prior intravenous thrombolysis & $145(26.4)$ & $368(30.5)$ & 9.3 & 0.07 & $115(28.3)$ & $102(25.1)$ & 7.2 & 0.30 \\
\hline Type of anesthesia & & & 16.8 & 0.01 & & & 4.1 & 0.55 \\
\hline Local anesthesia only & $265(48.2)$ & $500(41.5)$ & & & $190(46.7)$ & $184(45.2)$ & & \\
\hline Local anesthesia plus sedation & $92(16.7)$ & $190(15.8)$ & & & $68(16.7)$ & $60(14.7)$ & & \\
\hline General anesthesia & $193(35.1)$ & $515(42.7)$ & & & $149(36.6)$ & $163(40.1)$ & & \\
\hline Stent-retriever thrombectomy & $385(70.0)$ & $834(69.2)$ & 1.7 & 0.74 & $284(69.8)$ & $289(71.0)$ & 2.7 & 0.70 \\
\hline Aspiration thrombectomy & $14(2.6)$ & $40(3.3)$ & 4.6 & 0.38 & $13(3.2)$ & $15(3.7)$ & 2.7 & 0.70 \\
\hline Stent-retriever plus aspiration thrombectomy & $124(22.6)$ & $180(14.9)$ & 19.6 & $<0.01$ & $83(20.4)$ & $77(18.9)$ & 3.7 & 0.60 \\
\hline Pass number of thrombectomy, median (IQR) & $2(1-3)$ & $1(1,2)$ & 40.8 & $<0.01$ & $2(1-3)$ & $2(1-3)$ & 1.4 & 0.79 \\
\hline Emergency angioplasty/stenting & $45(8.2)$ & $471(39.1)$ & 78.1 & $<0.01$ & $45(11.1)$ & $57(14.0)$ & 8.9 & 0.20 \\
\hline Intra-arterial thrombolysis & $33(6.0)$ & $111(9.2)$ & 12.1 & 0.02 & $31(7.6)$ & $31(7.6)$ & 0.0 & 1.00 \\
\hline Intra-procedural use of tirofiban & $201(36.6)$ & $712(59.1)$ & 46.3 & $<0.01$ & $167(41.0)$ & $186(45.7)$ & 9.3 & 0.18 \\
\hline Intra-procedural use of heparin & $251(45.6)$ & $606(50.3)$ & 9.3 & 0.07 & $187(46.0)$ & $178(43.7)$ & 4.5 & 0.53 \\
\hline Onset-to-puncture time, median (IQR), min & $\begin{array}{l}260(195- \\
370)\end{array}$ & $\begin{array}{l}325(225- \\
484)\end{array}$ & 30.8 & $<0.01$ & $\begin{array}{l}284(200- \\
390)\end{array}$ & $\begin{array}{l}290(210- \\
410)\end{array}$ & 6.0 & 0.22 \\
\hline $\begin{array}{l}\text { Puncture-to-recanalization time, median (IQR), } \\
\text { min }\end{array}$ & $80(50-120)$ & $89(54-135)$ & 11.2 & 0.01 & $79(50-120)$ & $87(53-128)$ & 5.3 & 0.19 \\
\hline
\end{tabular}

Abbreviations: AF atrial fibrillation, ASPECTS Alberta Stroke Program Early CT Score, IQR interquartile range, mRS modified Rankin Scale, NIHSS National Institutes of Health Stroke Scale, pc-ASPECTS posterior circulation Alberta Stroke Program Early CT Score, SD standardized difference

Values are numbers with percentages in parentheses, unless indicated otherwise

${ }^{a}$ ASPECTS for anterior circulation stroke, and pc-ASPECTS for posterior circulation stroke

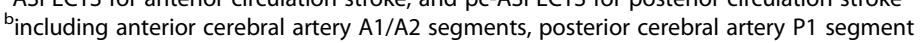




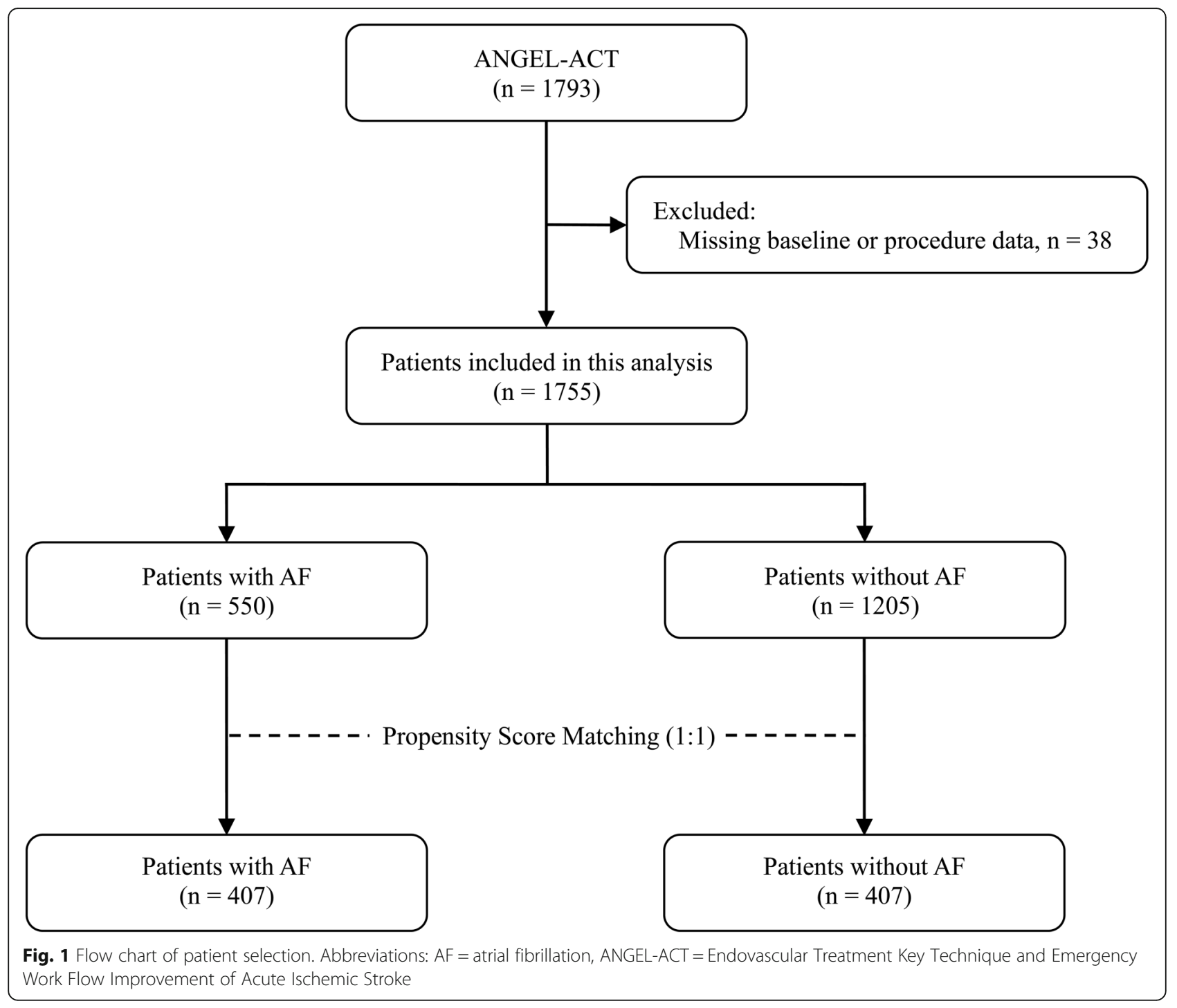

Comparisons of outcome measures between patients with and without AF were presented in Table 2. Before matching, there was no significant difference in recanalization rates between the two groups, but patients with AF had a higher 90-day mRS score $(P<0.01)$ and higher risks of intraprocedural complications $(P=0.02)$, hemorrhagic transformations within 24 hours (all $P<0.01$ ), and death within 90 days $(P=0.01)$, whereas they had lower proportions of mRS $0-1,0-2$, and $0-3$ points at 90 days (all $P<0.01$ ). After matching, the difference in the primary outcome - 90-day mRS score no longer existed between patients with and without AF (median: 3 vs. 3 points; $P=0.29$ ). In addition, all differences in secondary and safety outcomes that differed between both groups before matching also disappeared.

\section{Discussion}

This real-world registry study in China found that patients with AF were older, had more severe symptoms on admission, a lower proportion of posterior circulation occlusions, and a shorter time from onset to puncture. After matching for baseline characteristics using propensity scores, AF was not independently associated with 90-day functional outcomes, recanalization rates, and intra-procedural complications.

A subgroup analysis of the MR CLEAN trial (Multicenter Randomized Clinical Trial of Endovascular Treatment for Acute Ischemic Stroke in the Netherlands) showed a trend towards a decreased treatment effect of EVT in patients with AF. However, the sample size of AF patients in their study was rather small, thus no definite conclusion could be drawn [16]. A subsequent meta-analysis from the HERMES collaboration (Highly 
Table 2 Outcome measures of patients with AF versus without AF

\begin{tabular}{|c|c|c|c|c|c|c|c|c|}
\hline \multirow[t]{3}{*}{ Outcome variables } & \multicolumn{4}{|c|}{ Pre-matched population $(n=1755)$} & \multicolumn{4}{|c|}{ Post-matched population $(n=814)$} \\
\hline & \multirow{2}{*}{$\begin{array}{l}\text { With AF } \\
(n=550)\end{array}$} & \multirow{2}{*}{$\begin{array}{l}\text { Without AF } \\
(n=1205)\end{array}$} & \multicolumn{2}{|c|}{ Univariable analysis } & \multirow{2}{*}{$\begin{array}{l}\text { With AF } \\
(n=407)\end{array}$} & \multirow{2}{*}{$\begin{array}{l}\text { Without AF } \\
(n=407)\end{array}$} & \multicolumn{2}{|c|}{ Univariable analysis } \\
\hline & & & $\begin{array}{l}\text { Effect size } \\
(95 \% \mathrm{Cl})\end{array}$ & P-value & & & $\begin{array}{l}\text { Effect size } \\
(95 \% \mathrm{Cl})\end{array}$ & $P$-value \\
\hline \multicolumn{9}{|l|}{ Primary outcome } \\
\hline $\mathrm{mRS}$ at $90 \mathrm{~d}$, median (IQR) & $4(1-5)$ & $3(0-5)$ & $0.59(0.47-0.74)^{a}$ & $<0.01$ & $3(0-5)$ & $3(1-5)$ & 1. $16(0.82-1.52)^{a}$ & 0.29 \\
\hline \multicolumn{9}{|l|}{ Secondary outcomes } \\
\hline $\begin{array}{l}\text { Successful } \\
\text { recanalization after first } \\
\text { attempt }^{c}\end{array}$ & $267 / 550(48.6)$ & $588 / 1205(48.8)$ & $0.99(0.81-1.21)^{b}$ & 0.92 & 209/407 (51.4) & 190/407 (46.7) & 1. $21(0.92-1.59)^{b}$ & 0.18 \\
\hline $\begin{array}{l}\text { Successful } \\
\text { recanalization after final } \\
\text { attempt }^{c}\end{array}$ & $479 / 550(87.1)$ & 1065/1205 (88.4) & $0.89(0.65-1.20)^{b}$ & 0.44 & $355 / 407(87.2)$ & $347 / 407(85.3)$ & $1.18(0.79-1.76)^{b}$ & 0.42 \\
\hline $\begin{array}{l}\text { Complete } \\
\text { recanalization after final } \\
\text { attempt }^{d}\end{array}$ & $376 / 550(68.4)$ & $789 / 1205(65.5)$ & 1. $14(0.92-1.41)^{\mathrm{b}}$ & 0.24 & 279/407 (68.6) & 264/407 (64.9) & $1.18(0.88-1.58)^{b}$ & 0.27 \\
\hline mRS $0-1$ at $90 \mathrm{~d}$ & $174 / 518(33.6)$ & $521 / 1162(44.8)$ & $0.62(0.50-0.77)^{b}$ & $<0.01$ & 143/387 (37.0) & $143 / 386(37.1)$ & $1.00(0.74-1.33)^{b}$ & 0.98 \\
\hline mRS $0-2$ at $90 \mathrm{~d}$ & 195/518 (37.6) & $565 / 1162(48.6)$ & $0.64(0.52-0.79)^{b}$ & $<0.01$ & $160 / 387(41.3)$ & $155 / 386(40.2)$ & $1.05(0.79-1.40)^{b}$ & 0.74 \\
\hline mRS $0-3$ at $90 \mathrm{~d}$ & $252 / 518(48.7)$ & $676 / 1162(58.2)$ & $0.68(0.55-0.84)^{b}$ & $<0.01$ & 208/387 (53.8) & 192/386 (49.7) & $1.17(0.89-1.56)^{b}$ & 0.27 \\
\hline \multicolumn{9}{|l|}{ Safety outcomes } \\
\hline $\begin{array}{l}\text { Intra-procedural } \\
\text { complications }^{e}\end{array}$ & $63 / 550(11.5)$ & 95/1205 (7.9) & $1.51(1.08-2.12)^{b}$ & 0.02 & 43/407 (10.6) & 36/407 (8.8) & 1. $22(0.76-1.94)^{b}$ & 0.41 \\
\hline Any ICH within $24 \mathrm{~h}$ & $158 / 516(30.6)$ & $222 / 1163(19.1)$ & $1.87(1.48-2.37)^{\mathrm{b}}$ & $<0.01$ & 106/384 (27.6) & 95/388 (24.5) & 1. $18(0.85-1.62)^{b}$ & 0.32 \\
\hline $\mathrm{PH} 2$ within $24 \mathrm{~h}^{\mathrm{f}}$ & $35 / 516(6.8)$ & $41 / 1163(3.5)$ & $1.99(1.25-3.17)^{b}$ & $<0.01$ & $25 / 384(6.5)$ & 23/388 (5.9) & $1.11(0.62-1.98)^{b}$ & 0.74 \\
\hline $\begin{array}{l}\text { Symptomatic ICH } \\
\text { within } 24 \mathrm{~h}^{f}\end{array}$ & $54 / 513(10.5)$ & $70 / 1156(6.1)$ & $1.83(1.26-2.65)^{b}$ & $<0.01$ & $36 / 381(9.4)$ & $35 / 386(9.1)$ & $1.05(0.64-1.71)^{b}$ & 0.86 \\
\hline Death within $90 \mathrm{~d}$ & $100 / 518(19.3)$ & $162 / 1162(13.9)$ & $1.48(1.12-1.94)^{b}$ & 0.01 & 63/387 (16.3) & $71 / 386(18.4)$ & $0.86(0.59-1.25)^{b}$ & 0.44 \\
\hline
\end{tabular}

Abbreviations: AF atrial fibrillation, $C I$ confidence interval, ICH intracranial hemorrhage, IQR interquartile range, $m R S$ modified Rankin Scale, $m T I C I$ modified Thrombolysis in Cerebral Infarction, $\mathrm{OR}$ odds ratio, $\mathrm{PH} 2$ parenchymal hematoma type 2

Data are shown as the event number/total number (\%), unless otherwise indicated

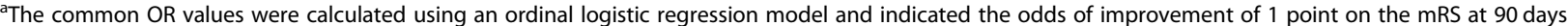

${ }^{\mathrm{b}}$ The OR values were calculated using a binary logistic regression model

'Defined as $\mathrm{mTICl}$ of $2 \mathrm{~b}-3$

${ }^{\mathrm{d}}$ Defined as $\mathrm{mTICl}$ of 3

e Including new territorial embolization, arterial perforation, arterial dissection, vasospasm requiring treatment and in-stent thrombosis

fccording to the Heidelberg Bleeding Classification

Effective Reperfusion Evaluated in Multiple Endovascular Stroke Trials) demonstrated no interaction between $\mathrm{AF}$ and functional outcomes after EVT, but found a trend towards a lower rate of symptomatic ICH in AIS patients with $\mathrm{AF}$ (3.4\% in AF patients vs. $4.5 \%$ in nonAF patients), which might be related to the lower percentage of pre-treatment with IVT $(76.3 \%$ in AF patients vs. $90.6 \%$ in non-AF patients). This is probably mainly due to the fact that patients with AF are more likely to taking oral anticoagulants, which is a contraindication for the administration of tPA [17]. Conversely, a posthoc analysis of a multi-center head-to-head clinical trial revealed that $\mathrm{AF}$ was an independent risk factor for any $\mathrm{ICH}$ in AIS patients undergoing stent-retriever thrombectomy, which was partly attributable to the adjusted anticoagulation status and more retrieval attempts by mediation analyses [18]. Furthermore, a national registry study assessing post-thrombectomy outcomes found no difference in either in-hospital or discharge outcomes between matched patients with or without AF, [19] whereas two other studies suggested faster procedural time, fewer passes, higher rates of first pass effect, successful reperfusion and good functional outcome with AF-related stroke [20, 21].

Previous observations found patients with AIS caused by AF tend to have more bleedings and worse outcomes after EVT than those without AF $[16,18]$. However, special cautions should be taken when interpreting these results, such a statement could lead to misconclusions to suspecting or even denying EVT to patients with AF. We may expect that AIS caused by a sudden embolus from the cardiovascular circulation can progress faster than AIS caused by progressive carotid or intracranial artery stenosis, where there may be time for 
development of collaterals [26]. In this study, patients with AF were treated about 1 hour earlier (median time from onset to puncture: $260 \mathrm{~min}$ vs. $325 \mathrm{~min}$ ) compared to those without AF, suggesting a faster infarct growth rate and a stronger time dependence of reperfusion therapy in AF-related stroke.

Strengths of this study were the large sample size of enrolled patients $(n=1755)$ and the high prevalence of AF (31. 3\%), resulting in more reliable estimations. Also, comparison of outcomes after PSM was a strength. Finally, all radiological and clinical outcomes in this analysis were centrally adjudicated by the independent imaging core laboratory or clinical events committee, except those intra-procedural complications were locally scored by site investigators. Nevertheless, our study has some limitations. First, the collateral status has been shown to be an excellent predictor of stroke outcomes, [27] so a major limitation of this study is the lack of assessment of collateral status, which has been postulated as a possible reason for difference in functional outcomes post-EVT of LVO patients with vs. without AF $[28,29]$. Second, this study was conducted in Chinese population, where the prevalence of intracranial atherosclerotic disease (ICAD) is very high [30]. In this context, an underlying ICAD stenotic lesion is often cited as a possible reason for immediate re-occlusion after thrombectomy that results in bailout intracranial angioplasty or stenting, thus potentially having an impact on the outcomes [31]. Our findings should be interpreted with caution and could not easily be extrapolated to other populations. Third, patients with AF may have more comorbidities (e.g., decreased ejection fraction, valvular heart disease, other organ failure), larger infarct core, and different texture of thrombus compared to those without AF. However, these variables were not collected in the ANGEL-ACT registry, so their confounding effects could not be ruled out. Finally, no information on antithrombotic therapy from post-procedure to discharge, treatment adherence and rehabilitation training after discharge was recorded, therefore limiting comments on the association between them and functional outcomes.

\section{Conclusion}

The present study found no difference in the radiological and clinical outcomes following EVT between Chinese AIS patients with and without AF, implying AF status should not hamper the decision making to proceed to EVT. Furthermore, our results were in contrast to the increased hemorrhage rates and worse functional outcomes observed in AF-related stroke treated with supportive care or IVT. It is known that thrombolysis is less used in patients with AF-related LVO and, if used, has only limited effect. Thus, the fact is EVT might be the best chance for these patients.

\section{Abbreviations}

AF: Atrial fibrillation; AIS: Acute ischemic stroke; ANGEL-ACT: Endovascular Treatment Key Technique and Emergency Work Flow Improvement of Acute Ischemic Stroke; ASPECTS: Alberta Stroke Program Early CT Score;

Cl: Confidence interval; EVT: Endovascular treatment; HERMES: Highly Effective Reperfusion Evaluated in Multiple Endovascular Stroke Trials; ICH: Intracranial hemorrhage; IQR: Interquartile range; IVT: Intravenous thrombolysis; LVO: Large vessel occlusion; MR CLEAN: Multicenter Randomized Clinical Trial of Endovascular Treatment for Acute Ischemic Stroke in the Netherlands; mRS: Modified Rankin Scale; mTICl: Modified Thrombolysis in Cerebral Infarction; NIHSS: National Institutes of Health Stroke Scale; OR: Odds ratio; pc-ASPECTS: Posterior circulation Alberta Stroke Program Early CT Score; PH2: Parenchymal hematoma type 2;

PSM: Propensity score matching; SD: Standardized difference

\section{Acknowledgements}

We thank all participating hospitals, relevant clinicians, statisticians, and imaging and laboratory technicians.

The ANGEL-ACT study group

Zhongrong Miao'; Liqiang Gui ${ }^{8}$; Cunfeng Song ${ }^{9}$; Ya Peng ${ }^{10}$; Jin $\mathrm{Wu}^{11}$; Shijun $Z^{12}$; Junfeng Zhao ${ }^{13}$; Zhiming Zhou ${ }^{14}$; Yongli Li ${ }^{15}$; Ping Jing ${ }^{16}$; Lei Yang ${ }^{17}$; Yajie Liu ${ }^{18}$; Qingshi Zhao ${ }^{19}$; Yan Liu ${ }^{20}$; Xiaoxiang Peng ${ }^{21}$; Qingchun Gao ${ }^{22}$; Zaiyu Guo ${ }^{23}$; Wenhuo Chen ${ }^{24}$; Weirong $\mathrm{Li}^{25}$; Xiaojiang Cheng ${ }^{26}$; Yun $\mathrm{Xu}^{27}$; Yongqiang Zhang ${ }^{28}$; Guilian Zhang ${ }^{29}$; Yijiu Lu ${ }^{30}$; Xinyu Lu $^{31}$; Dengxiang Wang ${ }^{32}$; Yan Wang ${ }^{33}$; Hao Li ${ }^{34}$; Yang Hua ${ }^{35}$; Deqin Geng ${ }^{36}$; Haicheng Yuan ${ }^{37}$; Hongwei Wang ${ }^{38}$; Haihua Yang ${ }^{39}$; Zengwu Wang ${ }^{40}$; Liping Wei ${ }^{41}$; Xuancong Liufu $^{42}$; Xiangqun Shi ${ }^{43}$; Juntao $\mathrm{Li}^{44}$; Wenwu Yang ${ }^{45}$; Wenji Jing ${ }^{46}$; Xiang Yong $^{47}$; Leyuan Wang ${ }^{48}$; Chunlei $\mathrm{Li}^{49}$; Yibin Cao ${ }^{50}$; Qingfeng $\mathrm{Zhu}^{51}$; Peng Zhang ${ }^{52}$; Xiang Luo ${ }^{53}$; Shengli Chen ${ }^{54}$; WenWu Peng ${ }^{55}$; Lixin Wang ${ }^{56}$; Xue Wen $^{57}$; Shugui Shi ${ }^{58}$; Wanming Wang ${ }^{59}$; Wang Bo ${ }^{60}$; Pu Yuan ${ }^{61}$; Dong Wang $^{62}$; Haitao Guan ${ }^{63}$; Wenbao Liang ${ }^{64}$; Daliang Ma ${ }^{65}$; Long Chen ${ }^{66}$; Yan $\mathrm{Xiao}^{67}$; Xiangdong $\mathrm{Xie}^{68}$; Zhonghua Shi ${ }^{69}$; Xiangjun Zeng ${ }^{70}$; Fanfan $\mathrm{Su}^{71}$; MingZe Chang ${ }^{72}$; Jijun Yin ${ }^{73}$; Hongxia Sun ${ }^{74}$; Chong $\mathrm{Li}^{75}$; Yong Bi ${ }^{76}$; Gang $\mathrm{Xie}^{77}$; Yuwu Zhao ${ }^{78}$; Chao Wang ${ }^{79}$; Peng Zhang ${ }^{80}$; Xianjun Wang ${ }^{81}$; Dongqun $\mathrm{Li}^{82}$; Hui Liang ${ }^{83}$; Zhonglun Chen ${ }^{84}$; Yan Wang ${ }^{85}$; Yuxin Wang ${ }^{86}$; Lin Yin ${ }^{87}$; HongKai Qiu ${ }^{88}$; Jun Wei ${ }^{89}$; Yaxuan Sun ${ }^{90}$; Xiaoya Feng ${ }^{91}$; Weihua Wu ${ }^{92}$; Lianbo $\mathrm{GaO}^{93}$; Zhibing Ai ${ }^{94}$; Lan Tan ${ }^{95}$; Li Ding ${ }^{96}$; Qilong Liang ${ }^{97}$; Zhimin Wang ${ }^{98}$; Jianwen Yang ${ }^{99}$; Ping $\mathrm{Xu}^{100}$; Wei Dong ${ }^{101}$; Quanle Zheng ${ }^{102}$; Zhenyun Zhu ${ }^{103}$; Liyue Zhao ${ }^{104}$; Qingbo Meng ${ }^{105}$; Yuqing Wei ${ }^{106}$; Xianglin Chen ${ }^{107}$; Wei Wang ${ }^{108}$; Dong Sun ${ }^{109}$; Yongxing Yan ${ }^{110}$; Guangxiong Yuan ${ }^{111}$; Yadong Yang ${ }^{112}$; Jianfeng Zhou'113; Zhi Yang ${ }^{114}$; Zhenzhong Zhang ${ }^{115}$; Ning Guan ${ }^{116}$; Huihong Wang ${ }^{117}$

'Beijing Tiantan Hospital, Beijing, China

${ }^{8}$ Langfang Changzheng Hospital, Hebei, China

'Liaocheng Third People's Hospital, Shandong, China

${ }^{10}$ The First People's Hospital of Changzhou, Jiangsu, China

${ }^{11}$ The Second Affiliated Hospital of Nanjing Medical University, Jiangsu, China

${ }^{12}$ Fengrun District People's Hospital of Tangshan City, Hebei, China

${ }^{13}$ SiPing Central People's Hospital, Jilin, China

${ }^{14}$ Yijishan Hospital of Wannan Medical College, Anhui, China

${ }^{15}$ The 2nd Affiliated Hospital of Harbin Medical University, Heilongjiang, China

${ }^{16}$ The Central Hospital of Wuhan, Hubei, China

${ }^{17}$ The First Hospital of Shijiazhuang, Hebei, China

${ }^{18}$ Shenzhen Hospital of Southern Medical University, Guangdong, China

${ }^{19}$ The People's Hospital of Longhua, Guangdong, China

${ }^{20}$ Jingjiang People's Hospital, Jiangsu, China

${ }^{21}$ The Third People's Hospital of Hubei Province, Hubei, China

${ }^{22}$ The Second Affiliated Hospital of Guangzhou Medical University, Guangdong, China

${ }^{23}$ Tianjin TEDA Hospital, Tianjin, China

${ }^{24}$ Zhangzhou Affiliated Hospital of Fujian Medical University, Fujian, China

${ }^{25}$ Taiyuan Central Hospital, Shanxi, China

${ }^{26}$ The First Affiliated Hospital of Xinjiang Medical University, Xinjiang, China

${ }^{27}$ Affiliated Drum Tower Hospital of Nanjing University Medical School,

Jiangsu, China

${ }^{28}$ The First People's Hospital of Wenling, Zhejiang, China

${ }^{29}$ The Second Affiliated Hospital of Xi'an Jiaotong University, Shaanxi, China 
${ }^{30}$ The First People's Hospital of Yulin, Guangxi, China

${ }^{31}$ Zhenjiang First People's Hospital, Jiangsu, China

${ }^{32}$ Qitaihe Coal General Hospital Heilongjiang, China

${ }^{33}$ People's Hospital of Tangshan City, Hebei, China

${ }^{34}$ Affiliated Hospital of Guilin Medical University, Guangxi, China

${ }^{35}$ The Affiliated Hospital of Guizhou Medical University, Guizhou Province, China

${ }^{36}$ The Affiliated Hospital of Xuzhou Medical University, Jiangsu, China

${ }^{37}$ Qingdao Central Hospital, Shandong, China

${ }^{38}$ The Fourth People's Hospital of Langfang City, Hebei, China

${ }^{39}$ Beijing Daxing hospital, Beijing, China

${ }^{40}$ Weifang People's Hospital, Shandong, China

${ }^{41}$ Luoyang General Hospital Affiliated to Zhengzhou University, Henan, China

${ }^{42}$ Dongguan Kanghua Hospital, Guangdong, China

${ }^{43}$ Shunde Hospital of Southern Medical University, Guangdong, China

${ }^{44}$ Handan Central Hospital, Hebei, China

${ }^{45}$ The 981 hospital of the Chinese People's Liberation Army, Hebei, China

${ }^{46}$ Linfen people's Hospital, Shanxi, China

${ }^{47}$ Anshun people's Hospital of Guizhou, China

${ }^{48}$ Changle People's Hospital, Shandong, China

${ }^{49}$ The Second People's Hospital of Dongying, Shandong, China

${ }^{50}$ Tangshan Gongren hospital, Hebei, China

${ }^{51}$ PLA 985 Hospital of the Joint Logistics Support Force, Shanxi, China

${ }^{52}$ Gaomi People's Hospital, Shandong, China

${ }^{53}$ Tongji Hospital, Tongji Medical College, Huazhong University of Science and Technology, Hubei, China

${ }^{54}$ Chongqing Sanxia Center Hospital, Chongqing, China

${ }^{55} \mathrm{Hospital}$ of Traditional Chinese Medicine of Qiannan, Guizhou, China

${ }^{56}$ Guangdong Hospital of Chinese Medicine, Guangdong, China

${ }^{57}$ People's hospital of Yangjiang, Guangdong, China

${ }^{58}$ The Third Affiliated Hospital of CQMU, Chongqing, China

${ }^{59}$ General Hospital of The Yangtze River Shipping, Hubei, China

${ }^{60}$ First People's Hospital of Bijie City, Guizhou, China

${ }^{61}$ Suqian People's Hospital of Nanjing Drum-Tower Hospital Group, Jiangsu,

China

${ }^{62}$ Weifang TCM Hospital, Shandong, China

${ }^{63}$ The Third Affiliated Hospital of Guangzhou Medical University, Guangdong,

China

${ }^{64}$ Karamay Central hospital, Xinjiang, China

${ }^{65}$ The third people's Hospital of Xinjiang Uygur Autonomous Region,

Xinjiang, China

${ }^{66}$ Wulanchabu City Central Hospital, Inner Mongolia, China

${ }^{67}$ Hospital of Xinjiang Production \& Construction Corps, Xinjiang, China

${ }^{68}$ Jiaozuo Second people's hospital, Henan, China

${ }^{69}$ The 904 Hospital of Joint Logistic Support Force of PLA, Jiangsu, China

${ }^{70}$ Ganzhou People's Hospital, Jiangxi, China

${ }^{71}$ The 967 Hospital of the Joint Logistics Support Force of PLA, Liaoning,

China

${ }^{72}$ The Affiliated Hospital of Northwest University Xi'an No.3 Hospital, Shaanxi, China

${ }^{73}$ The Second Hospital of Liao Cheng, Shandong, China

${ }^{74} \mathrm{Jilin}$ Province People's Hospital, Jilin, China

${ }^{75}$ People's Hospital of Huanghua City, Hebei, China

${ }^{76}$ Shanghai Forth People's Hospital, Shanghai, China

${ }^{77}$ Wanbei Coal-electricity Group General Hospital, Anhui, China

${ }^{78}$ Shanghai Jiao Tong University Affiliated Sixth People's Hospital, Shanghai,

China

${ }^{79}$ Binzhou Medical University Hospital, Shandong, China

${ }^{80}$ The 988 hospital of the people's liberation army, Henan, China

${ }^{81}$ Linyi People's Hospital, Shandong, China

${ }^{82}$ Yingkou City Central Hospital, Liaoning, China

${ }^{83}$ Yantaishan Hospital, Shandong, China

${ }^{84}$ Mianyang Central hospital, Sichuan, China

${ }^{85}$ Chengdu Fifth People's Hospital, Sichuan, China

${ }^{86}$ Hengshui Fifth Hospital of Heng shui City, Hebei, China

${ }^{87}$ Second Hospital of Dalian Medical University, Liaoning, China

${ }^{88}$ Boai Hospital of Zhongshan, Guangdong, China

${ }^{89}$ The First People's Hospital of Yibin, Sichuan, China

${ }^{90}$ Shanxi provincial people's hospital, Shanxi, China

${ }^{91}$ Shandong Provincial Third Hospital, Cheeloo College of Medicine,

Shandong University, Shandong, China
${ }^{92}$ Chuxiong State People's Hospital, Chuxiong, Yunnan, China

${ }^{93}$ The Fourth Affiliated Hospital of China Medical University, Liaoning, China

${ }^{94}$ Taihe Hospital, Shiyan, Hubei, China

${ }^{95}$ Qingdao Municipal Hospital, Shandong, China

${ }^{96}$ The First People's Hospital of Yunnan Province, Yunnan, China

${ }^{97}$ The NO.2 People's Hospital of Lanzhou, Gansu, China

${ }^{98}$ Taizhou First People's Hospital, Zhejiang, China

${ }^{99}$ Hunan Provincial People's Hospital, Hunan, China

${ }^{100}$ First People's Hospital of Changde City, Hunan, China

${ }^{101}$ Zhejiang Yuyao People's Hospital, Zhejiang, China

${ }^{102}$ Aidebao Hospital, Hebei, China

${ }^{103}$ The First Hospital of Fangshan District, Beijing, China

${ }^{104}$ Tianjin Xiqing Hospital, Tianjin, China

${ }^{105}$ People's Hospital of Zunhua, Hebei, China

${ }^{106}$ Xingtai Third Hospital, Hebei, China

${ }^{107}$ Qingyuan People's Hospital, Guangdong, China

${ }^{108}$ Fengcheng City Central Hospital, Liaoning, China

${ }^{109}$ People's Hospital of Hejian City, Hebei, China

${ }^{110}$ Hangzhou Third People's Hospital, Zhejiang, China

${ }^{111}$ Xiangtan Central Hospital, Hunan, China

${ }^{112}$ People's Hospital of Nanpi Country, Hebei, China

${ }^{113}$ Liuzhou Railway Central Hospital, Guangxi, China

${ }^{114}$ Maoming People's Hospital, Guangdong, China

${ }^{115}$ Tongde Hospital of Zhejiang Province, Zhejiang, China

${ }^{116}$ The First Affiliated Hospital of Jinzhou Medical University, Liaoning, China

${ }^{117}$ Xishan coal electricity group worker general hospital, Shaanxi, China

\section{Authors' contributions}

$X T, D M$ and $Z M$ designed the study; $X T, S L$ and $W L$ wrote the main manuscript text and prepared figures; $Z R$ and $R L$ made the critical revision of manuscript; AW performed the statistical analysis; BJ, XZ, XH, GL and GM conducted the study and acquired the data; YW, YW and ZM supervised the study; DM and ZM analyzed and interpreted the data. All authors reviewed the manuscript. The author(s) read and approved the final manuscript

\section{Funding}

This study was funded by the National Key Research and Development Program of China (2018YFC1312801, 2016YFC1301500), China Postdoctoral Science Foundation (2019 M650773). The funding body did not play any role in the design of the study and collection, analysis, and interpretation of data and in writing the manuscript.

\section{Availability of data and materials}

The data that support the findings of this study are available from the corresponding author Dapeng Mo (bjttmodp@163.com) or Zhongrong Miao (zhongrongm@163.com) upon reasonable request.

\section{Declarations}

\section{Ethics approval and consent to participate}

The protocol was approved by the ethics committees of Beijing Tiantan Hospital and each participating site. Each participant or his/her representative gave written informed consent before being enrolled in the study. The study procedures were in accordance with the 1964 Helsinki declaration and its later amendments.

\section{Consent for publication}

Not applicable.

\section{Competing interests}

The authors have no financial conflicts of interest.

\section{Author details}

${ }^{1}$ Department of Interventional Neuroradiology, Beijing Tiantan Hospital, Capital Medical University, No.119 South 4th Ring West Road, Fengtai District, Beijing, China. ${ }^{2}$ Department of General Practice, Beijing Mentougou District Hospital, Beijing, China. ${ }^{3}$ Center for Medical Device Evaluation, National Medical Product Administration, Beijing, China. ${ }^{4}$ Department of Neurosurgery, University of South Florida, Tampa, Florida, USA. ${ }^{5}$ Beijing Institute of Brain Disorders, Capital Medical University, Beijing, China. ${ }^{6}$ China National Clinical Research Center for Neurological Diseases, Beijing Tiantan 
Hospital, Capital Medical University, Beijing, China. ${ }^{7}$ Department of Neurology, Beijing Tiantan Hospital, Capital Medical University, Beijing, China.

\section{Received: 6 June 2021 Accepted: 28 August 2021}

\section{Published online: 29 September 2021}

\section{References}

1. January CT, Wann LS, Calkins H, Chen LY, Cigarroa JE, Cleveland JC Jr, et al, 2019 AHA/ACC/HRS focused update of the 2014 AHA/ACC/HRS guideline for the Management of Patients with Atrial Fibrillation: a report of the American College of Cardiology/American Heart Association task force on clinical practice guidelines and the Heart Rhythm Society. J Am Coll Cardiol. 2019;74(1):104-32. https://doi.org/10.1016/j.jacc.2019.01.011.

2. Goyal M, Demchuk AM, Menon BK, Eesa M, Rempel JL, Thornton J, et al. Randomized assessment of rapid endovascular treatment of ischemic stroke. N Engl J Med. 2015;372:1019-30.

3. Campbell BC, Mitchell PJ, Kleinig TJ, Dewey HM, Churilov L, Yassi N, et al. Endovascular therapy for ischemic stroke with perfusion-imaging selection N Engl J Med. 2015;372(11):1009-18. https://doi.org/10.1056/NEJMoa1414 792.

4. Berkhemer OA, Fransen PS, Beumer D, van den Berg LA, Lingsma HF, Yoo $A J$, et al. A randomized trial of intraarterial treatment for acute ischemic stroke. N Engl J Med. 2015;372:11-20.

5. Jovin TG, Chamorro A, Cobo E, de Miquel MA, Molina CA, Rovira A, et al Thrombectomy within 8 hours after symptom onset in ischemic stroke. N Engl J Med. 2015:372(24):2296-306. https://doi.org/10.1056/NEJMoa1503780.

6. Saver JL, Goyal M, Bonafe A, Diener HC, Levy El, Pereira VM, et al. Stentretriever thrombectomy after intravenous t-PA vs. t-PA alone in stroke. N Engl J Med. 2015;372(24):2285-95. https://doi.org/10.1056/NEJMoa1415061.

7. Nogueira RG, Jadhav AP, Haussen DC, Bonafe A, Budzik RF, Bhuva P, et al. Thrombectomy 6 to 24 Hours after Stroke with a Mismatch between Deficit and Infarct. N Engl J Med. 2018;378:11-21.

8. Albers GW, Marks MP, Kemp S, Christensen S, Tsai JP, Ortega-Gutierrez S, et al. Thrombectomy for stroke at 6 to 16 hours with selection by perfusion imaging. N Engl J Med. 2018;378(8):708-18. https://doi.org/10.1056/ NEJMoa1713973.

9. Lin S, Wu B, Hao ZL, Kong FY, Tao WD, Wang DR, et al. Characteristics, treatment and outcome of ischemic stroke with atrial fibrillation in a Chinese hospital-based stroke study. Cerebrovasc Dis. 2011;31(5):419-26. https://doi.org/10.1159/000323221.

10. Kimura K, Minematsu K, Yamaguchi T. Atrial fibrillation as a predictive factor for severe stroke and early death in 15,831 patients with acute ischaemic stroke. J Neurol Neurosurg Psychiatry. 2005;76(5):679-83. https://doi.org/1 0.1136/jnnp.2004.048827.

11. Henninger N, Goddeau RP Jr, Karmarkar A, Helenius J, McManus DD. Atrial fibrillation is associated with a worse 90-day outcome than other Cardioembolic stroke subtypes. Stroke. 2016;47(6):1486-92. https://doi.org/1 0.1161/STROKEAHA.116.012865.

12. Steger C, Pratter A, Martinek-Bregel M, Avanzini M, Valentin A, Slany J, et al. Stroke patients with atrial fibrillation have a worse prognosis than patients without: data from the Austrian stroke registry. Eur Heart J. 2004;25(19): 1734-40. https://doi.org/10.1016/j.ehj.2004.06.030.

13. Yue R, Li D, Yu J, Li S, Ma Y, Huang S, et al. Atrial Fibrillation is Associated With Poor Outcomes in Thrombolyzed Patients With Acute Ischemic Stroke: A Systematic Review and Meta-Analysis. Medicine (Baltimore). 2016;95: e3054.

14. Hu Y, Ji C. Efficacy and safety of thrombolysis for acute ischemic stroke with atrial fibrillation: a meta-analysis. BMC Neurol. 2021;21(1):66. https://doi.org/1 0.1186/s12883-021-02095-x

15. Powers WJ, Rabinstein AA, Ackerson T, Adeoye OM, Bambakidis NC, Becker K, et al. Guidelines for the Early Management of Patients With Acute Ischemic Stroke: 2019 update to the 2018 guidelines for the early Management of Acute Ischemic Stroke: a guideline for healthcare professionals from the American Heart Association/American Stroke Association. Stroke. 2019;50(12):e344-418. https://doi.org/10.1161/STR 0000000000000211.

16. Heshmatollah A, Fransen P, Berkhemer OA, Beumer D, van der Lugt A Majoie C, et al. Endovascular thrombectomy in patients with acute ischaemic stroke and atrial fibrillation: a MR CLEAN subgroup analysis. Eurolntervention. 2017;13(8):996-1002. https://doi.org/10.4244/EIJ-D-1600905.
17. Smaal JA, de Ridder IR, Heshmatollah A, van Zwam WH, Dippel D, Majoie CB, et al. Effect of atrial fibrillation on endovascular thrombectomy for acute ischemic stroke. A meta-analysis of individual patient data from six randomised trials: results from the HERMES collaboration. Eur Stroke J. 2020; 5(3):245-51. https://doi.org/10.1177/2396987320923447.

18. Huang K, Zha M, Gao J, Du J, Liu R, Liu X. Increased intracranial hemorrhage of mechanical thrombectomy in acute ischemic stroke patients with atrial fibrillation. J Thromb Thrombolysis. 2021;51(2):536-44. https://doi.org/10.1 007/s11239-020-02269-3.

19. Munir MB, Alqahtani F, Beltagy A, Tarabishy A, Alkhouli M. Comparative outcomes of mechanical Thrombectomy for acute ischemic stroke in patients with and without atrial fibrillation. J Vasc Interv Radiol. 2017;28(11): 1604-5. https://doi.org/10.1016/j.jvir.2017.06.024.

20. Akbik F, Alawieh A, Cawley CM, Howard BM, Tong FC, Nahab F, et al. Differential effect of mechanical thrombectomy and intravenous thrombolysis in atrial fibrillation associated stroke. J Neurointerv Surg. 2021; 13(10):883-8. https://doi.org/10.1136/neurintsurg-2020-016720.

21. Lin CJ, Luo CB, Chien C, Chang FC, Lin CJ, Lee IH, et al. Better endovascular mechanical thrombectomy outcome in atrial fibrillation patients with acute ischemic stroke: a single-center experience. J Chin Med Assoc. 2020;83(8): 756-60. https://doi.org/10.1097/JCMA.0000000000000377.

22. Jia B, Ren Z, Mokin M, Burgin WS, Bauer CT, Fiehler J, et al. Current Status of Endovascular Treatment for Acute Large Vessel Occlusion in China: A RealWorld Nationwide Registry. Stroke. 2021;52:STROKEAHA120031869.

23. Zaidat OO, Yoo AJ, Khatri P, Tomsick TA, von Kummer R, Saver $J$, et al. Recommendations on angiographic revascularization grading standards for acute ischemic stroke: a consensus statement. Stroke. 2013;44(9):2650-63. https://doi.org/10.1161/STROKEAHA.113.001972.

24. von Kummer R, Broderick JP, Campbell BC, et al. The Heidelberg bleeding classification: classification of bleeding events after ischemic stroke and reperfusion therapy. Stroke. 2015;46(10):2981-6. https://doi.org/10.1161/ STROKEAHA.115.010049.

25. Tong $X$, Wang $Y$, Fiehler J, Bauer $C T$, Jia B, Zhang $X$, et al. Thrombectomy Versus Combined Thrombolysis and Thrombectomy in Patients With Acute Stroke: A Matched-Control Study. Stroke. 2021;52:STROKEAHA120031599.

26. Widimsky P. Acute ischaemic stroke in atrial fibrillation: worse outcomes unrelated to treatment methods. Eurolntervention. 2017;13(8):905-6. https:// doi.org/10.4244/EIJV1318A134.

27. Tan BY, Kong WY, Ngiam JN, Teoh HL, Sharma VK, Yeo LL. The role of topographic collaterals in predicting functional outcome after thrombolysis in anterior circulation ischemic stroke. J Neuroimaging. 2017;27(2):217-20. https://doi.org/10.1111/jon.12387.

28. Sun B, Shi Z, Pu J, Yang S, Wang H, Yang D, et al. Effects of mechanical thrombectomy for acute stroke patients with etiology of large artery atherosclerosis. J Neurol Sci. 2019;396:178-83. https://doi.org/10.1016/j.jns.2 018.10.017.

29. Guglielmi V, LeCouffe NE, Zinkstok SM, Compagne K, Eker R, Treurniet KM, et al. Collateral circulation and outcome in atherosclerotic versus Cardioembolic cerebral large vessel occlusion. Stroke. 2019;50(12):3360-8. https://doi.org/10.1161/STROKEAHA.119.026299.

30. Wang Y, Zhao X, Liu L, Soo YO, Pu Y, Pan Y, et al. Prevalence and outcomes of symptomatic intracranial large artery stenoses and occlusions in China: the Chinese intracranial atherosclerosis (CICAS) study. Stroke. 2014;45(3): 663-9. https://doi.org/10.1161/STROKEAHA.113.003508.

31. Yeo L, Bhogal P, Gopinathan A, Cunli Y, Tan B, Andersson T. Why does mechanical Thrombectomy in large vessel occlusion sometimes fail? : A review of the literature. Clin Neuroradiol. 2019;29(3):401-14. https://doi.org/1 0.1007/s00062-019-00777-1.

\section{Publisher's Note}

Springer Nature remains neutral with regard to jurisdictional claims in published maps and institutional affiliations. 